\title{
A Study of Synchronization Algorithms in OFDM/WiMAX Systems
}

\author{
Erick R. Sousa, Karlo G. Lenzi, Luís G.P. Meloni
}

\begin{abstract}
A review of OFDM synchronization algorithms applied to IEEE 802.16 standard is presented. An extended Morelli algorithm is proposed and compared with classical synchronization methods: Schmidl and Tufvesson (Matched-Filter). The timing metric of that extended algorithm is close to matchedfilter timing detection. The frequency offset is calculated using a BLUE approach, resulting in an estimation range of $[-G / 2, G / 2]$ subcarriers, where $G$ is the number of repeated patterns in the preamble. The detection probabilities in Rice, Rayleigh and static ISI channels is also simulated, and interesting results are obtained for each algorithm studied.
\end{abstract}

Keywords-OFDM, WiMAX, software defined radio, synchronization, FPGA.

\section{INTRODUCTION}

Time detection of arriving symbols and estimation of the carrier frequency offset (CFO) between transmitter and receiver is the starting point - and probably the most important point - of a reliable communication system. OFDM modulation technique is known to be sensitive to CFO [1], as the frequency offset causes loss of orthogonality among subcarriers, causing inter-carrier interference (ICI).

In the last years, many advances have been achieved on the OFDM synchronization techniques. Many of these advances were possible by the use of preambles with special features. Some of these advances still cannot be used in practice, because they are not yet included in any standard body. For example, the works in [2], [3] and [4], achieved better detection characteristics over Schmidl algorithm basically by doing changes in the preamble as well as some minor modifications on the timing metric.

Considering the preambles in IEEE 802.16 systems [5] as a fixed system setting, only a few algorithms can be explored. These are mainly prior-1999 algorithms, such as Schmidl [6], Morelli [7] and Tufvesson [8] algorithms. Schmidl uses a 2part preamble and calculates the time and frequency offsets between transmitter and receiver. This algorithm expands the theory developed by Moose [9]. Morelli algorithm considers that the signal is synchronized in time a priori, then calculates the frequency offset using a Best-LinearUnbiased-Estimator (BLUE) approach. Tufvesson's method uses a matched filter for timing synchronization, and calculates the frequency offset using a Maximum-Likelihood estimate similar to Schmidl/Moose method. The ExtendedMorelli algorithm proposed in this work is a joint-detection

Erick R. Sousa, Karlo G. Lenzi, Luís G.P. Meloni, Laboratório de Processamento Digital de Sinais em Tempo Real (RT-DSP), Departamento de Comunicações, Faculdade de Engenharia Elétrica e Computação, Unicamp, Campinas, Brasil. Emails: \{erick,lenzi,meloni\}@decom.fee.unicamp.br algorithm that uses a Maximum-Likelihood timing estimation along with a BLUE frequency offset estimation.

Also, in order to make calculations less complex, most algorithms consider the channel as Additive-White-Gaussian. In general, this is not the case in real communication systems, so it is important to compare the algorithms' behaviour under other channel types, such as Rice, Rayleigh and cable channels.

\section{SYSTEM DESCRIPTION}

OFDM signals are generated by Inverse Fast Fourier Transform (IFFT) of a set of subsymbols $\left\{X_{i}\right\}$, which are modulated in BPSK, QPSK, QAM, or any other modulation technique, following the equation:

$$
x(k)=\frac{1}{\sqrt{N}} \sum_{i=0}^{N-1} X_{i} e^{j 2 \pi i k / N},
$$

where $N$ represents the number of subcarriers, $x(k)$ is the time-domain OFDM signal that has a duration of $T_{s}$ seconds, and lenght of $N$ samples.

Before transmission, $x(k)$ is enlarged by $L$ samples, also known as "cyclic prefix". The last $L$ samples are copied to the start of the symbol, generating a $N+L$ length symbol. The cyclic prefix must be longer than the channel impulse response, in order to mitigate inter-symbol-interference (ISI).

At the receiver, the signal is sampled with frequency $N / T_{s}$ Hertz, and a frequency offset $v$ is also added. This frequency offset is normalized in respect to the subcarrier spacing $1 / T_{s}$. The received signal can be expressed in the form:

$$
r(k+\theta)=s(k+\theta) e^{j 2 \pi v k / N}+w(k+\theta),
$$

where $w(k)$ is white Gaussian noise with zero mean and power $\sigma_{w}^{2}=E\left\{|w(k)|^{2}\right\}, \theta$ is the time delay between transmitter and receiver. Also, $s(k)$ is defined as:

$$
s(k)=\frac{1}{\sqrt{N}} \sum_{i=0}^{N-1} H_{i} X_{i} e^{j 2 \pi i k / N},
$$

where $H_{i}$ is the digital frequency-domain channel response. The received signal power is defined as $\sigma_{s}^{2}=E\left\{|s(k)|^{2}\right\}$, so the signal-to-noise-ratio can be expressed as $\mathrm{SNR}=\sigma_{s}^{2} / \sigma_{w}^{2}$.

The synchronization is performed by the aid of a preamble with $G$ equal parts. This preamble can be synthesized sending pseudo-noise sequences on the subcarriers with index multiple of $G$, and setting zero on the remaining subcarriers. In IEEE 802.16 systems, a preamble with $G=4$ equal parts (P4x64) is described [5]. 


\section{Estimation Method}

The proposed estimation method can be considered an extension of Morelli's Algorithm [7]. Morelli's algorithm considers the received signal $r(k)$ to be prior synchronized in time, so the aid of another synchronization method is necessary, adding up the receiver complexity.

In fact, the correlation method used in [7] resembles others synchronization algorithms such as Schmidl [6] and Vande-beek [10] methods. By making some adjustments in the calculations presented in [7], one can obtain both time and CFO estimation, without increasing the complexity too much. This method will be referred as Extended Morelli algorithm, or E-Morelli from now on.

\section{A. Timing estimation}

The received signal must be delayed and self-correlated according to the equation:

$$
R_{m}(k)=2 \sum_{i=k}^{k+m N / G+L-1} \frac{r(i) r^{*}(i+N-m N / G)}{m N / G+L},
$$

the energy can be calculated by:

$$
E_{m}(k)=\rho \sum_{i=k}^{k+m N / G+L-1} \frac{\left(|r(i)|^{2}+|r(i+N-m N / G)|^{2}\right)}{m N / G+L},
$$

and the m-th correlation:

$$
\lambda_{m}(k)=\left|R_{m}(k)\right|-E_{m}(k),
$$

for $0 \leq m \leq G-1$.

Equation 6 is in fact the Van-de-Beek ML-estimation metric [10]. When $m=0$, correlation is performed between cyclic prefix and the last $L$ samples of the symbol; the Equation 6 is reduced to Van-de-Beek Maximum-Likelihood timing metric:

$$
\lambda_{\mathrm{VdB}}(k)=2\left|\sum_{i=k}^{k+L-1} r_{k} r_{k+N}^{*}\right|-\rho \sum_{i=k}^{k+L-1}\left(\left|r_{k}\right|^{2}+\left|r_{k+N}\right|^{2}\right) \text {. }
$$

The overall E-Morelli timing metric can be defined as:

$$
\lambda_{\mathrm{T}}(k)=\sum_{m=0}^{G-1} \lambda_{m}(k),
$$

and the delay estimation $\hat{\theta}$ can be obtained by:

$$
\hat{\theta}=\arg \max \left\{\lambda_{\mathrm{T}}(k)\right\} .
$$

The time estimation error can be defined as $\Delta \theta=\theta-\hat{\theta}$. When $-L<\Delta \theta<0$, the synchronization performed within the limits of the cyclic prefix, and just some phase offset is added in the received signal. When $\hat{\theta}$ falls outside the cyclic prefix range, the signal will suffer interference and therefore poor performance is achieved [11].

The main difference between Morelli and E-Morelli correlations is the increased length of the moving sums, now $L$ samples longer. This increase generates a sharp edge on the timing metric, which reduces time estimation variance. The set of correlations $\left\{R_{m}(k)\right\}$ can explore the repetitive nature of the P4x64 preamble pattern defined in IEEE 802.16, therefore
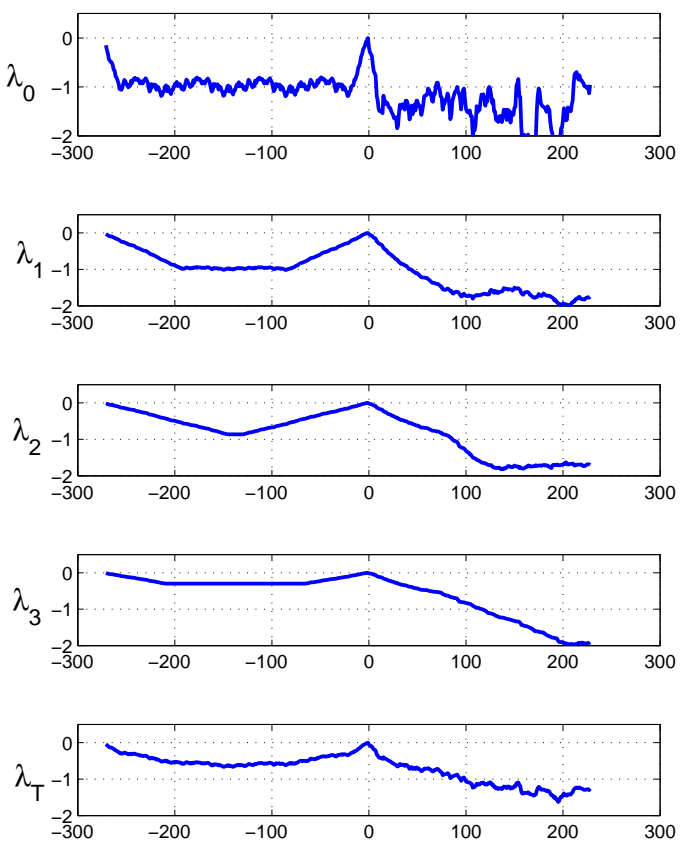

Fig. 1. Extended Morelli Correlations

calculating four correlations with different window lenghts: $L$ for $\lambda_{0},(N / 4+L)$ for $\lambda_{1},(N / 2+L)$ for $\lambda_{2},(3 N / 4+L)$ for $\lambda_{3}$. A realization of the explained set of correlations is shown in Fig. 1.

\section{B. CFO estimation}

The CFO is estimated using a BLUE estimator based on correlation performed in Equation 4. This method is similar to Morelli's correlation in [7], and produces the same results in respect to estimator variance and error. The $\mathrm{CFO}$ estimation can be obtained by

$$
\hat{v}=\frac{G}{2} \frac{1}{\pi} \sum_{m=1}^{H} w(m) \phi(m)
$$

where

$$
\phi(m)=\left[\angle R_{m}(\hat{\theta})-\angle R_{m-1}(\hat{\theta})\right]_{2 \pi}
$$

for $1 \leq m \leq H$. The weights $w(m)$ are calculated as shown in [7]:

$$
w(m)=3 \frac{(G-m)(G-m+1)-H(G-H)}{H\left(4 H^{2}-6 G H+3 G^{2}-1\right)} .
$$

The CFO estimator range is $\left[-\frac{G f_{s}}{2 N}, \frac{G f_{s}}{2 N}\right]$ Hertz, where $f_{s}=$ $N / T_{s}$ is the sampling frequency. This CFO estimator is the same estimator of Morelli Algorithm in [7], consequently it achieves the same Cramer-Rao low bound and variance. 


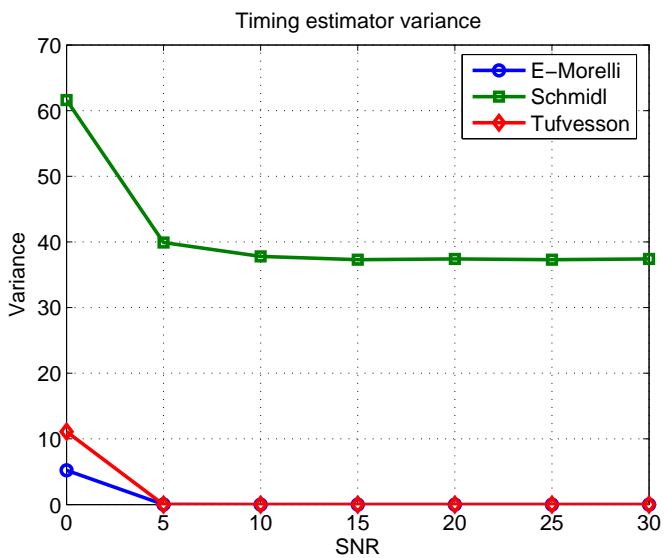

Fig. 2. Variance of timing estimator.

\section{Simulation Results}

In this section, we compare three algorithms: Schmidl [6], Tufvesson [8] and E-Morelli. In each SNR point, 10,000 realizations were performed, in order to extract some important statistics, such as timing and CFO estimation variance, and detection probabilities under four different channels.

\section{A. Timing Estimator Statistics}

One important feature in synchronization algorithms is the timing estimator variance. The estimator's variance must be low enough in order the signal detection occurs inside the cyclic prefix. Another important feature is the probability of detection: the algorithm must be robust enough to detect the incoming packets even under bad channel conditions.

In Figure 2, each SNR point was produced after 10.000 realizations of each algorithm. We can see that the variance of Schmidl algorithm [6] does not tend to zero as SNR increases. This result was expected, as the timing metric for this algorithm reaches a plateau of maximum length $L$. The Tufvesson[8] and E-Morelli algorithms tend to zero variance as SNR increases. This represents a more stable behaviour in the timing estimate.

\section{B. Frequency Estimator Statistics}

Another important point in synchronization is the frequency estimation variance. A high variance means higher residual error that the next processing stages in the receiver must cope with. In Fig. 3, we can see that E-Morelli method has the lower estimator variance. Schmidl and Tufvesson aproaches have virtually equal variances.

In Figs. 3, 4 and 5, we can see that when using AWGN, static ISI an Rice channels, the variances tend to decay as SNR increases. But in Fig. 6, when simulating under Rayleigh channel, the variances reach a floor and do not tend to decay as SNR increases.

\section{Detection Probabilities}

This metric can be defined as:

$$
P_{d}=P\{-L \leq \Delta \theta \leq 0\}
$$

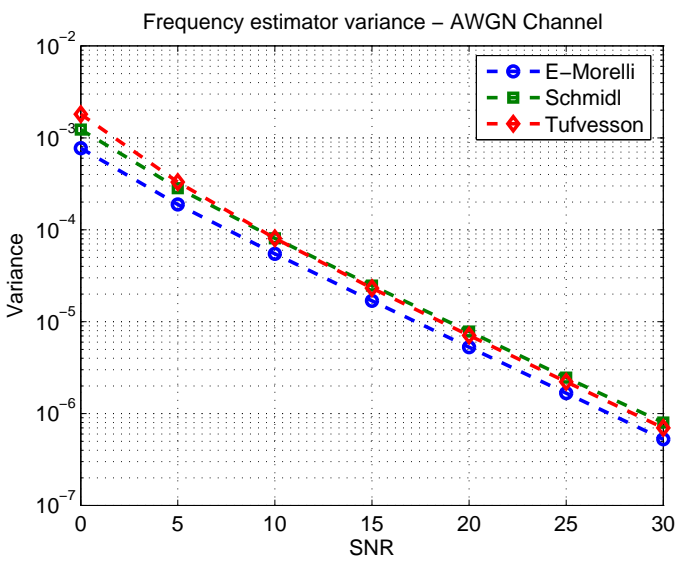

Fig. 3. Variance of CFO estimator under AWGN channel.

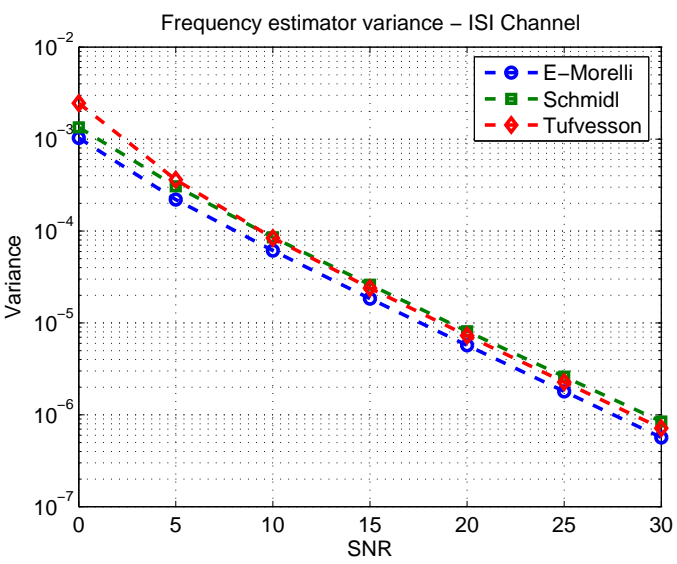

Fig. 4. Variance of CFO estimator under static ISI channel.

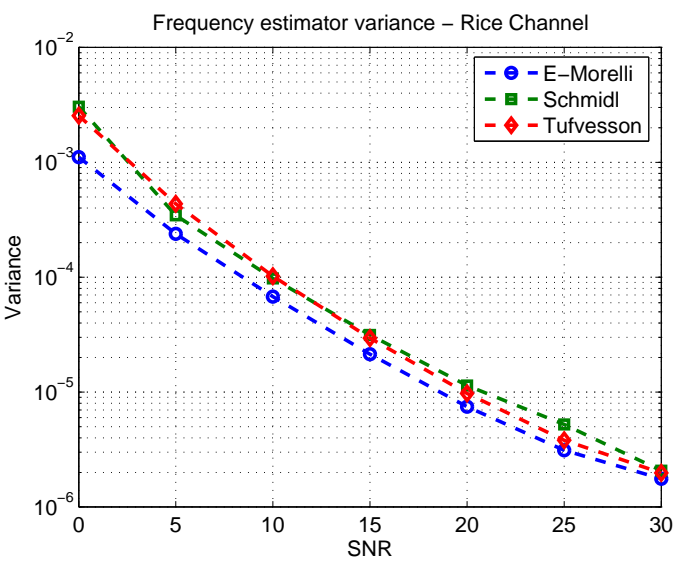

Fig. 5. Variance of CFO estimator under Rice channel.

as $\Delta \theta$ is a random discrete variable, we can write:

$$
P\{-L \leq \Delta \theta \leq 0\}=\sum_{i=-L}^{0} P\{\Delta \theta=i\} .
$$

The probabilities $P\{\Delta \theta=i\}$ can be estimated by simulation of histograms, so a close value of $P_{d}$ can be achieved.

The variable $\Delta \theta$ depends on the algorithm tested, as well as channel characteristics. Some algorithms generate a lower 


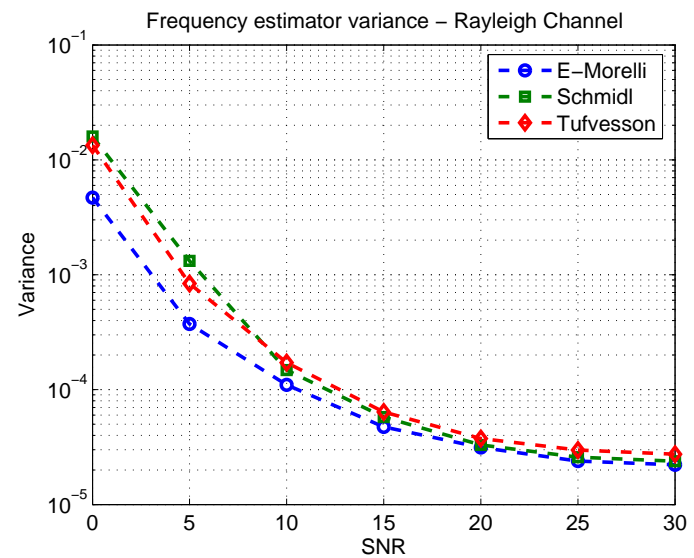

Fig. 6. Variance of CFO estimator under Rayleigh channel.

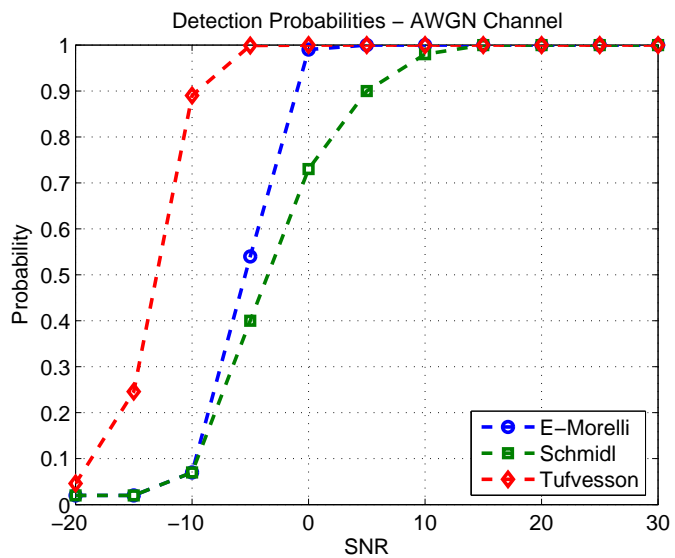

Fig. 7. Detection Probabilities for AWGN channel.

variance $\Delta \theta$, while some channels tend to raise the variance of $\Delta \theta$. In the next sections, we study four channels: AWGN, static ISI, Rice, and Rayleigh channels.

1) Detection in AWGN Channel: In Fig. 7, estimation of detection probabilities in a SNR range of $[-20 \mathrm{~dB}, 20 \mathrm{~dB}]$ is shown. The algorithm that performed best was the Tufvesson[8], followed by E-Morelli and Schmidl [6]. This result was expected, as the matched filter is widely used in telecommunications as an optimum method for signal detection under AWGN.

The probability of detection only points out how much an algorithm is capable of detecting an incoming packet, and does not assure that the frequency estimate is good enough for reliable communication. In fact, although some algorithms are able to detect preambles under negative SNRs, few communication systems operate in such an extreme interference region.

2) Detection in an ISI Channel: a simple static ISI channel was simulated as a $10^{\text {th }}$ order low-pass FIR. The low-pass characteristic was choosen because many transmission lines present this behaviour. The channel frequency response is illustrated in Fig. 8. The simulations were performed using a 16-sample cyclic prefix, hence the first 10 samples of the cyclic prefix are distorted by ISI, and 6 samples are free of interference. The results are presented in Fig. 9.

In this type of channel, higher frequencies suffer a larger

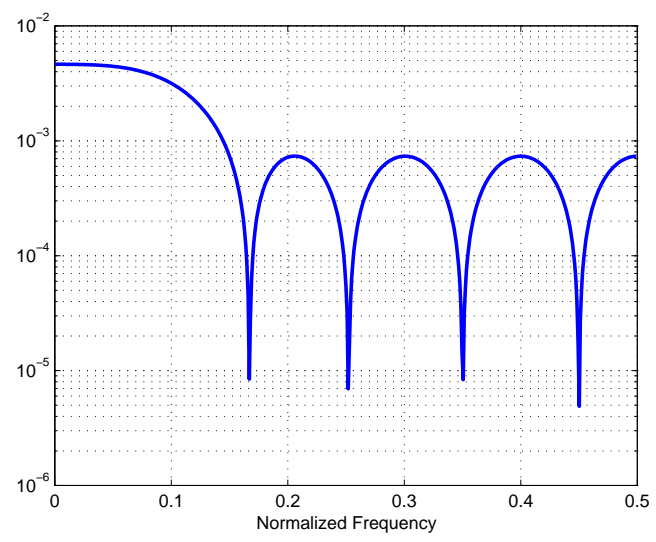

Fig. 8. Static ISI channel transfer function.

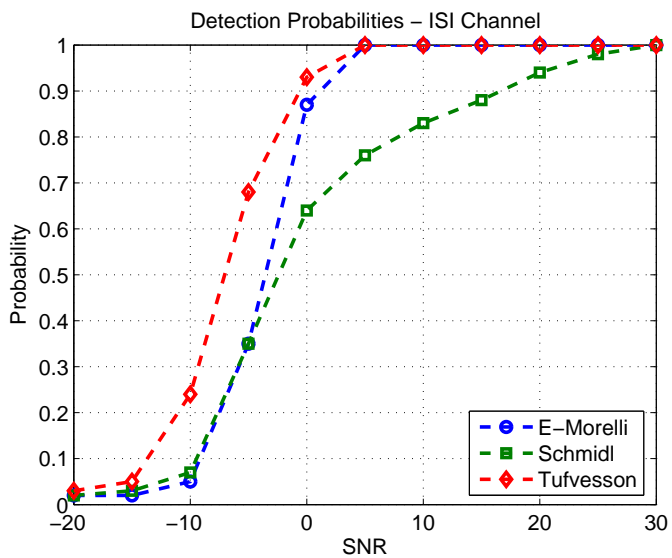

Fig. 9. Detection Probabilities for static ISI channel.

distortion due to white noise which is added after filtering. A sharp fall in performance is noticed, because the higher frequencies of the received preamble are distorted. The Tufvesson method is not optimum anymore, and also suffers degradation: it reached $100 \%$ detection in $5 \mathrm{~dB}$ of SNR, but in the pure AWGN channel, reached this point around $-5 \mathrm{~dB}$. The matched filter is not "matched" anymore with the signal being received, so this loss in performance is expected.

3) Detection in Rice Channel: The Ricean channel used here is described in [12]. This is a line-of-sight channel, which means a portion of the signal travels through a direct path and another portion through a difuse path. It was also added a Doppler spread of $100 \mathrm{~Hz}$. The model used for simulation can be found in [13].

The curves for detection probability in Fig. 10 resemble the ones for AWGN channel. When a large portion of the signal travels by the direct path, the channel behaviour tends to AWGN. When the inverse occurs, it tends to behave as a Rayleigh channel. In this simulation, we used a ratio $K \approx 16$ between the direct signal power and the difuse signal power.

4) Detection in Rayleigh Channel: The channel simulated here is described in [12]; a typical urban Rayleigh non-lineof-sight channel. A Doppler frequency of $100 \mathrm{~Hz}$ was added as well. The channel profile is detailed in Table I.

In Fig. 11, we can see that the detection probability for 


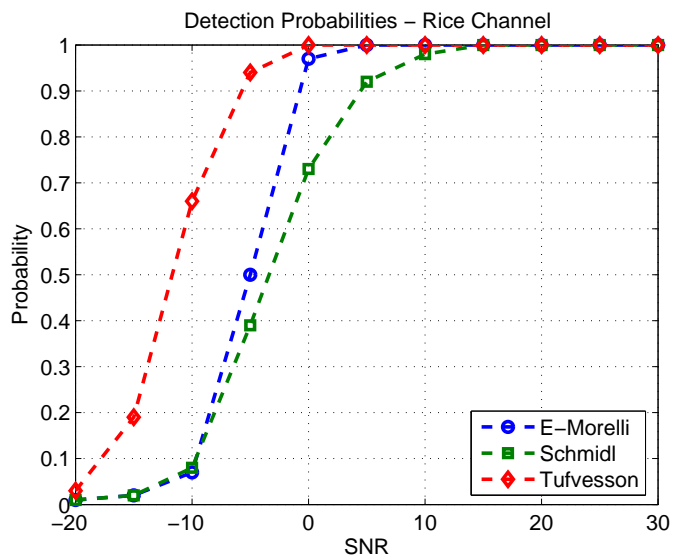

Fig. 10. Detection Probabilities for Rice channel.

TABELA I

RAYLEIGH CHANNEL GAIN PROFILE.

\begin{tabular}{|c||c|c|}
\hline Tap & Delay $(\mu s)$ & Average Power $(\mathrm{dB})$ \\
\hline \hline$g_{0}$ & 0 & -3.5366 \\
\hline$g_{1}$ & 0.5 & -4.4428 \\
\hline$g_{2}$ & 1.0 & -11.6020 \\
\hline$g_{3}$ & 1.5 & -10.2797 \\
\hline$g_{4}$ & 2.0 & -14.7055 \\
\hline
\end{tabular}

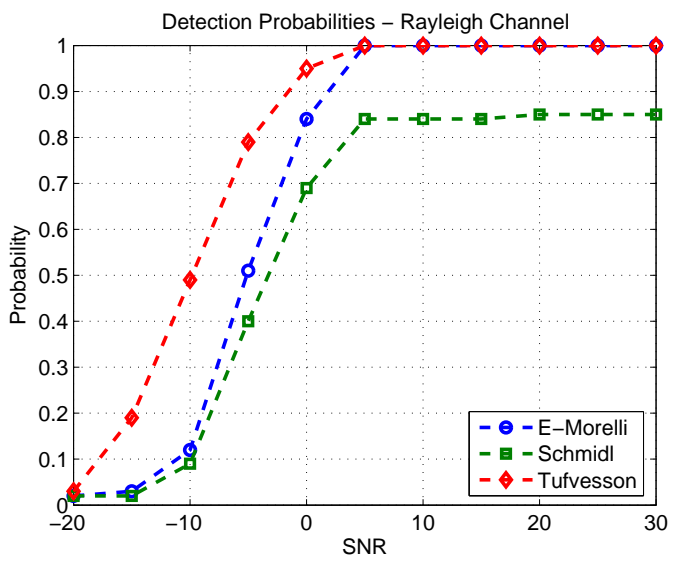

Fig. 11. Detection Probabilities for Rayleigh channel.

Schmidl algorithm reaches a maximum plateau. This happens because even in high SNRs, this channel can exhibit relatively high signal to interference ratio (SIR), due to multipath reflections and fading.

Another interpretation for Schmidl's algorithm behaviour is that, under Rayleigh channel, the instantaneous SNR drops occur frequently - the so-called fading effect -. When the channel is in a deep SNR drop, the additive noise is so high that timing and frequency estimates become poor. This effect tends to affect the final result, even if the average SNR is increased. It is important to note that increasing the average SNR does not change how frequently the instantaneous SNR drops, as the fading is controlled by Doppler frequency.

The other algorithms react better than Schmidl because they have better performance under higher additive noise. So even under an instantaneous SNR drop, E-Morelli or Tufvesson algorithms are able to react better than Schmidl.

\section{CONCLUSION}

Considering the algorithms compared in this work, in general, the Tufvesson approach has the best timing estimate, while E-Morelli has the best frequency offset estimate. In fact, E-Morelli timing estimate is close to the Tufvesson estimate, in order that no difference would be noticed between both, under usual SNR conditions.

Most systems operate under fair SNR conditions; in this case, E-Morelli would show the same performance as the Tufvesson in the time estimate, but better performance in frequency estimate, e.g. lower variance and larger frequency acquisition range. For the channels simulated in this work, if SNR $>5 \mathrm{~dB}$ both algorithms reach $100 \%$ detection probability.

In general, we conclude that if good SNR occurs, E-Morelli algorithm is a better choice when compared to Tufvesson and Schmidl.

\section{ACKNOWLEDGEMENTS}

The authors would like to thank financial support by FINEP Project $n^{\circ} 6341$.

\section{REFERENCES}

[1] T. Pollet, M. Van Bladel, Ber sensitivity of ofdm systems to carrier frequency offset and wiener phase noise. IEEE Transactions on Communications, 43:191193, 1995.

[2] Zhongshan Zhang, Keping Long, Joint frame synchronization and frequency offset estimation in ofdm systems. IEEE Transactions on Broadcasting, 51:389394, 2005.

[3] Seung Duk Choi, Jung Min Choi, An initial timing offset estimation method for ofdm systems in rayleigh fading channel. IEEE, 2006.

[4] H. Minn, M. Zeng, On timing offset estimation for ofdm systems. IEEE Communications Letters, 4:242244, 2000.

[5] 802.16 IEEE Standard for Local and metropolitan area networks, 2004.

[6] Timothy M. Schmidl and Donald C. Cox, Robust Frequency and Timing Synchronization for OFDM. IEEE Transactions on Communications, VOL 45, No 12, December 1997.

[7] Michelle Morelli and Umberto Mengali, An Improved Frequency Offset Estimator for OFDM Applications. IEEE Communications Letters, VOL 3, No 3, March 1999.

[8] Fredrik Tufvesson, Ove Edfors and Mike Faulkner, Time and Frequency Synchronization for OFDM using PN-Sequence Preambles. IEEE VTC'99.

[9] P. Moose, A technique for orthogonal frequency-division multiplexing frequency offset correction. IEEE Transactions on Communications, vol 10, 2908-2914, 1994.

[10] Jan-Jaap van de Beek and Magnus Sandell, Low Complex Frame Synchronization in OFDM Systems. Proc. of Intern. Conf. on Universal Personal Comm. ICUPC'95, November 1995.

[11] Tilde Fusco, Synchronization Technics for OFDM Systems. PhD Thesis, Universita Degli Studi Di Napoli Federico II, 2004.

[12] 3GPP TR 25.943; Deployment Aspects, 2002-2006.

[13] Michel C. Jeruchim, Philip Balaban and K. Sam Shanmugan, Simulation of Communication Systems. Kluwer, 2002. 